\title{
Une prise industrielle dans une nappe souterraine
}

\section{An industrial offtake for underground water}

\author{
IPR A. BOURGIN \\ INGHNIEUR HN CHEF DES PONTS ET CHAUSSÉES
}

La Houille Blanche a déjà décrit certains types exceptionnels de prises d'eau, barrages sous-glaciaires ou barrages sur une rivière souterraine. Il nous paraît intéressant de présenter à ses lecteurs un nouvel exemple d'un type peu habituel, celui d'un captage d'une nappe dans un but industriel.

Certes, il est banal de capter, pour les besoins d'un hameau, d'un village, d'une ville même, les quelques litres, les quelques dizaines de litres nécessaires à son alimentation, en aménageant, par puits ou galeries drainantes, une source ì l'émergence d'une nappe. Grenoble, par exemple, tire d'un réseau de drains, situés dans la nappe de Rochefort, dépendant du Drac et de la Gresse, plus de $1.000 \mathrm{l} / \mathrm{s}$.

Nous connaissons l'exemple d'un canal agricole, alimenté à partir d'une nappe. En Champsaur, dans la vallée du Haut-Drac, de nombreux canaux d'irrigation dépendent de prises d'eau superficielles, précaires, exposées aux divagations perpétuelles du Drac, à ses crues. Les prises volantes imposent des frais annuels onéreux.

Le canal de Chabottes, au contraire, est alimenté par une galerie drainante dans les alluvions du Drac. Réalisée en 1910 sur des crédits d'Etat, elle est constituce par des buses de $0,80 \mathrm{~m}$ posées non jointives dans une tranchée et recoulvertes de pierraille.

Elle assure un débit de $400 \mathrm{l} / \mathrm{s}$ et alimente successivement le canal de Chabottes, le moulin Mouren et le canal de Saint-Bonnet. Il ne semble pas qu'aucun colmatage ait réduit les débits captés depuis l'origine. La régularité d'alimentation est précieuse.

Nous n'avions pas eu connaissance jusqu'à maintenant de captages de nappes réalisés dans un but industriel. II fallait pour cela que les débits soient élevés, la nappe suffisamment puissante, étendue, que sa disposition topographique crée la chute : or, les nappes sont rarement suspendues; ces dispositions se sont trouvées réunies au village de La Sone, en rive droite de l'Isère.

L'Isère, entre Saint-Gervais et Romans, coule dans une gorge peu profonde, taillée dans la mollasse miocène, recouverte par différentes terrasses : mindélienne, rissienne, wurmienne, etc.

La terrasse de Saint-Marcellin-Chatte est attribuée au riss. Limitée à l'ouest par la Combe Baudran, elle présente une surface de l'ordre de $10 \mathrm{~km}^{2}$, une puissance de $60 \mathrm{~m}$ au droit des gorges et recèle une nappe qui trouve son exutoire normal au contact des alluvions et de la mollasse, niveau argilo-calcaire, marne bleue attribuce au burdigalien m 2 et qui constitue le niveau imperméable.

De nombreuses sources ou suintements apparaissent donc en crête de la falaise, sur près de $2 \mathrm{~km}$ en amont de La Sone, et ont donné naissance à de fortes accumulations de tufs $\mathbf{A}_{t}$. Le village de La Sone, son église en particulier, sont fondés sur ce tuf.

Les captages de Chevrières, qui alimentent la ville de Saint-Marcellin, avec $50 \mathrm{l} / \mathrm{s}$, intéressent la zone amont de cette nappe. A La Sone, d'importantes sources, avec des débits de 25,50 ou $100 \mathrm{l} / \mathrm{s}$, ont alimenté, depuis des siècles, les industries locales, moulins et moulinages, au prix de travaux de collecte, d'amélioration, de captages sommaires, de galeries, exécutées de 1840 à 1865 (sources Pellerin, Arduin, Jubie, etc.). 
Un de ces meuniers, M. Griot, alerte vieillard de 80 ans, nous a fait visiter ses installations et tracé, avec ferveur et humour, l'historique de ses travaux. Mécontent des débits assurés par ses propres installations ou des droits d'eau acquis de ses voisins, il se décida à aller " chercher l'eau où elle est $»$. Partant de la cote 190 environ en bordure du GC 31, il fit creuser, de 1904 à 1908 , un réseau de galeries dont le développement atteint $300 \mathrm{~m}$. Il nous a décrit la technique employée. Forant au pic, puis à l'explosif (acide picrique acheté chez le marchand de couleurs) dans la marne bleue avec une couverture de quelques décimètres sous les alluvions aquifères, menacé à chaque instant par une venue d'eau catastrophique, il a réalisé par étapes une galerie de collecte maçonnée et un réseau de galeries drainantes plus ou moins ramifié. Les piédroits seuls ont été maçonnés, des tuyaux non jointifs disposés sur le radier, recouverts par des blocs de tuf, puis le toit a été foudroyé. Il a ainsi réalisé par une méthode originale la véritable percée d'une nappe par son fond imperméable.

Les galeries, actuellement noyées et siphonnantes, assurent un débit de 2501 en étiage, $300 \mathrm{l}$ en moyenne, $500 \mathrm{l}$ au maximum.

Les installations de chute sont scindées en deux. La chute amont (Laurent), de $18 \mathrm{~m}$, est équipée d'une turbine de $60 \mathrm{ch}$ munie d'un déchargeur. La chute aval (Griot), de $12 \mathrm{~m}$, d'une turbine de $40 \mathrm{ch}$ (Dumont 1926) et le canal de fuite souterrain restitue les eaux directement à l'Isère à la cote 158 environ.

Un pareil soutirage à la nappe n'a pu toutefois être réalisé sans influence sur les sources voisines. Il en est donc résulté, avec les industriels concurrents, d'interminables procès qui ne se sont terminés qu'en 1922. Il n'est pas de notre propos de résumer l'interminable procédure, ni d'en tirer les conséquences juridiques sur les droits à l'usage des eaux souterraines, basés sur les articles 552, 642 du Code Civil.

Les travaux de M. Griot ont entrainé deux procès intentés par des usiniers qui s'étaient vus privés d'eau; l'issue en a été différente. Dans le premier, le plaignant a été débouté par application de l'article 642 du Code Civil, une expertise ayant montré que les galeries drainantes étaient tracées dans le tréfonds des terrains appartenant à M. Griot où celui-ci avait droit de fouille.

Par contre, ce dernier a succombé dans la seconde instance et le dispositif appliqué est assez curieux. L'émergence tarie avait été acquise par les auteurs du propriétaire actuel, le vendeur de l'époque étant également propriétaire des terrains dominants; le tribunal a estimé que cette vente avait créé une servitude sur lesdits terrains, le vendeur n'ayant pu se réserver le droit de drainer l'eau dans son fond, ce qui aurait enlevé toute valeur à la chose vendue. Le tribunal a jugé que cette servitude était demeurée attachée au fond dominant el qu'elle s'appliquait à Griot, son actuel propriétaire.

Ces difficultés contentieuses ne nous empêcheront pas de saluer l'originalité technique de ces travaux et la persévérance de leur auteur.

\section{EXTRAITS DU CODE CIVII}

\section{ARTiGLE 552}

La propriété du sol emporte la propriété du dessus et du dessous.

Le propriétaire peut faire au-dessus toutes les plantations et constructions qu'il juge à propos, sauf les exceptions établies au titre des servitudes ou services fonciers.

Il peut faire au-dessous toutes les constructions et fouilles qu'il jugera à propos, et tirer de ces fouilles tous les produits qu'elles peuvent fournir, sauf les modifications résultant des lois et règlements relatifs aux mines. et des lois et règlements de police. Civ, 544, 553; 637 s., 641,671 s., $674.678,1859,2118$, 2133 ; L. 21 avril 1810.

\section{ARticle 642}

(Loi 8 avril 1898.) Celui qui a une source dans son fonds peut toujours user des eaux à sa volonté dans les limites et pour les besoins de son héritage.

Le propriétaire d'une source ne peut plus en user au préjudice des propriétaires des fonds inférieurs qui, depuis plus de trente ans, ont fait et terminé, sur le fonds où jaillit la source, des ouvrages apparents et permanents destinés à utiliser les eaux ou à en facilitèr le passage dans leur propriété.

Il ne peut pas non plus en user de manière à enlever aux habitants d'une commune, village ou hameau, l'eau qui leur est nécessaire; mais si les habitants n'en ont pas acquis ou prescrit l'usage, le propriétaire peut réclamer une indemnité, laquelle est réglée par experts.

\section{ARticle 1382}

Tout fait quelconque de l'homme qui cause à autrui un dommage, oblige celui-ci, par la faute duquel il est arrivé, à le réparer. 